\title{
Performances comparées du HDL-cholestérol et du ratio cholestérol total/HDL pour le dépistage du syndrome métabolique chez des adultes du Sud-Bénin (Afrique de l'Ouest)
}

\author{
Charles Jérôme SOSSA ${ }^{1,2^{*}}$, Victoire AGUEH ${ }^{1}$, Colette Sylvie AZANDJÈMÈ ${ }^{1,2}$, \\ Noel PARAÏSO ${ }^{1}$, Alphonse KPOZEHOUEN ${ }^{1}$, Hinson Antoine VIKKEY ${ }^{3}$, \\ Badirou AGUEMON ${ }^{3}$ et Hélène DELISLE ${ }^{2}$
}

\author{
${ }^{1}$ Institut Régional de Santé Publique (IRSP) de Ouidah, BP 384 Ouidah, Université d'Abomey Calavi, Bénin. \\ ${ }^{2}$ TRANSNUT, Centre Collaborateur OMS sur la Transition Nutritionnelle et le Développement, Département \\ de Nutrition, Faculté of Médecine, Université de Montréal. CP 6128 succ. Centre-ville, Montréal Qc, Canada, \\ H3C $3 J 7$. \\ ${ }^{3}$ Faculté des Sciences de la Santé de Cotonou, Université d'Abomey-Calavi, 01 BP 188 Cotonou. \\ *Auteur correspondant, E-mail : Sossajero@yahoo.com
}

\section{REMERCIEMENTS}

L'étude a été financée par l'Agence Canadienne de Développement International (ACDI) à travers le projet DFN (S064359).

\section{RESUME}

Les critères de définition du Syndrome métabolique (SMet) n'identifient pas convenablement les sujets d'origine africaine à risque. L'objectif était de déterminer lequel du ratio cholestérol total/HDL-cholestérol (CT/HDL-C) et du HDL-Cholestérol est le meilleur prédicteur du SMet chez les adultes béninois. L'étude de type transversal, issue d'une enquête longitudinale sur le risque cardiométabolique a inclu 416 sujets âgés de 29 à 69 ans. Les composantes du SMet considérées sont : l'obésité abdominale, la tension artérielle élevée, la glycémie élevée, le HDL-C bas et les triglycérides élevés. La comparaison des aires sous les courbes (AUC) de la «fonction d'efficacité du récepteur » (ROC) de prédiction de l'existence deux composantes du SMet autre que l'obésité abdominale, a permis de déterminer le meilleur prédicteur. Les prévalences du SMet étaient de 13,9\% selon la définition harmonisée, 12,3\% lorsque le HDL-C bas est remplacée par CT/HDL-C élevé. Les prévalences du HDL-bas et du CT/HDL-C élevé sont de 37,7\% et 22,6\%, respectivement. Pour le dépistage du SMet, l'AUC du CT/HDL-C est de 0,69 (IC 95\% 0,61-0,77) chez les femmes et 0,68 (IC 95\% 0,59-0,77) chez les hommes. L'AUC du HDL-C est de 0,45 (IC 95\% 0,37-0,53) chez les femmes et 0,40 (IC 95\% 0,30-0,44) chez les hommes. Le HDL-C et le CT/HDL-C ont une faible capacité prédictive pour le SMet, mais la composante CT/HDL-C prédit mieux le SMet que le HDL-C isolé. Toutefois, l'utilisation de l'un ou l'autre des deux paramètres ne modifie pas substantiellement la prévalence du SMet dans la population d'étude. (C) 2016 International Formulae Group. All rights reserved.

Mots clés : Syndrome métabolique, lipoprotéines, ratio CT/HDL-C, Sud-Bénin. 


\title{
Comparative performance of HDL-cholesterol and total cholesterol / HDL ratio for screening of metabolic syndrome in Southern Benin adults (West Africa)
}

\begin{abstract}
Current definition criteria of the metabolic syndrome (MetS) do not adequately identify at risk African origin subjects. The objective was to determine which of total cholesterol/HDL-cholesterol (TC/HDL-C) and HDL-cholesterol is the best predictor of metabolic syndrome (SMet) in Benin adults. This cross-sectional study was nested in a four-year follow-up study on cardiometabolic risk factors and included 416 adults aged 29-69 years. Components of MetS considered were abdominal obesity, high blood pressure (BP), high fasting glucose, low HDL-C and high triglycerides. Areas under the "Receiver operator characteristic" curves (AUC) for CT/HDL-C and HDL-C in predicting the presence of at least two other components of SMet were compared in order to determine the best predictor of SMet. The prevalence of SMet was $13.9 \%$, when replacing low HDL-C by high TC/HDL-C and $15.3 \%$ when both dyslipidemia indicators are combined. The prevalence of low HDL-C and high TC/HDL-C was $37.7 \%$ and $22.6 \%$, respectively $(\mathrm{p}<0.001)$. Screening for SMet, the AUC of TC/HDL-C were 0.69 (95\% CI 0.61-0.77) for women and 0.68 (95\% CI 0.59-0.77) in men. The AUC of HDL-C were 0.45 (95\% CI 0.37-0.53) for women and 0.40 (95\% CI 0.30-0.44) for men. Both TC/HDL-C and HDL-C showed some weak predictive values for SMet, but TC/HDL-C ratio predicted SMet better than HDL-C.
\end{abstract}

(C) 2016 International Formulae Group. All rights reserved.

Keywords: Metabolic syndrome, lipoprotein, ratio CT/HDL-C, Southern Benin.

\section{INTRODUCTION}

Le syndrome métabolique (Smet) ou syndrome d'insulino-résistance (Alberti et al., 2009) est caractérisé par la présence d'au moins trois quelconques des anomalies suivantes: tension artérielle élevée, faible concentration de lipoprotéines de haute densité cholestérol (HDL-C), triglycérides élevés, hyperglycémie et obésité abdominale. L'aptitude du SMet à prédire le risque cardiométabolique (RCM) a été abordée dans plusieurs études prospectives qui ont rapporté que sa présence était associée à un risque élevé de maladies cardiovasculaires (MCV) et de diabète de type 2 (Ford et al., 2002; Lorenzo et al., 2007 ; Després et al., 2008).

La prévalence du SMet est moins élevée chez les Afro-Américains que chez les Caucasiens alors que la prévalence des maladies cardiovasculaires (MCV) est plus élevée parmi les premiers (Sumner, 2009). Ce paradoxe est attribué aux différences ethniques entre les niveaux de triglycérides et de HDL-C entre les Afro-Américains et les Caucasiens. Parmi les individus Ouest
Africains avec SMet, moins de 25\% présentent des concentrations élevées de triglycérides (Sumner, 2010). Sossa et al.(2013a) ont rapporté que la prévalence du HDL-C bas était indépendante de l'insulinorésistance.

A l'inverse, le ratio Cholestérol total/HDL-C (CT/HDL-C) ou indice d'athérogénicité est un indicateur de RCM de valeur prédictive supérieure à celle des paramètres lipidiques isolés (Millan et al., 2009). Lorsque le cholestérol total, le HDL-C, le ratio $\mathrm{CT} / \mathrm{HDL}-\mathrm{Cl}$ sont comparés entre une population apparemment en bonne santé et celle ayant des antécédents d'infarctus du myocarde, le CT/HDL-C présente moins de superposition pour les deux populations (Millan et al., 2009). Cela illustre son pouvoir discriminant pour les maladies cardiovasculaires et probablement sa capacité prédictive du SMet.

L'objectif de l'étude était de déterminer lequel du ratio $\mathrm{CT} / \mathrm{HDL}$ et du HDL-C isolé prédit mieux le SMet chez des adultes béninois. L'hypothèse selon laquelle 
que le ratio $\mathrm{CT} / \mathrm{HDL}$ prédit mieux le SMet chez des adultes béninois que le HDL-C isolé a été formulée.

\section{MATERIEL ET METHODES \\ Cadre d'étude}

L'étude s'est déroulée au Sud du Bénin dans la grande métropole urbaine (Cotonou), en milieu semi urbain (ville de Ouidah) situé à 40 kilomètres de Cotonou et dans sa périphérie rurale.

\section{Population d'étude}

La population d'étude était constituée d'adultes béninois âgés de 29 à 69 ans.

\section{Type d'étude}

Il s'agissait d'une étude transversale nichée dans une étude longitudinale et observationnelle sur l'évolution sur une période de quatre ans des facteurs de RCM chez des adultes béninois. La présente étude exploite les données du dernier suivi de l'étude longitudinale.

\section{Echantillonnage}

\section{Taille de l'échantillon}

Parmi 541 sujets initialement inclus dans l'étude longitudinale (200 sujets en milieu urbain, 171 en milieu semi-urbain et 170 en milieu rural), un effectif de 416 sujets (208 femmes et 208 hommes) soit $77 \%$ ) ont été retrouvés au dernier suivi et ont été retenus pour le présent rapport. En fixant l'erreur de première espèce à $5 \%$ et celle de deuxième espèce à $20 \%$, l'aire sous la courbe $\mathrm{ROC}_{1}$ (HDL-C) à 0,55 et celle sous la courbe $\mathrm{ROC}_{2}$ (ratio CT/HDL-C) à 65\%, un effectif de 268 sujets est suffisant pour comparer les aires sous les deux courbes (AUC) «Receiver Operator Characteristics » (ROC).

\section{Sélection des sujets}

Les méthodes et techniques de sélection des sujets à l'entame de l'étude longitudinale dans chacun des trois sites ont été décrites en détails ailleurs (Ntandou et al., 2009). En bref, Les 416 sujets de la présente étude proviennent des participants de l'étude longitudinale observationnelle initiale qui a inclus des sujets apparemment en bonne santé sélectionnés aléatoirement par échantillonnage par grappes dans la plus grande ville $(n=200)$ de Cotonou, la petite ville de Ouidah $(\mathrm{n}=171)$, et la périphérie rurale de Ouidah $(\mathrm{n}=170)$ au Bénin. Parmi les 541 sujets initialement inclus dans l'étude longitudinale, un effectif de 416 ont été retrouvés au dernier suivi.

\section{Les variables de l'étude et leur mesure La variable dépendante}

La variable dépendante est la présence d'au moins deux composantes du SMet. Il s'agit de l'existence ou non d'au moins deux composantes quelconques (autres que le HDL$\mathrm{C}$ bas) parmi les quatre suivants utilisés pour définir le Smet: TA élevée, triglycérides élevés $\quad(>1,70 \mathrm{mmol} / \mathrm{L})$, hyperglycémie (glycémie $\geq 5,6 \mathrm{mmol} / \mathrm{L}$ et obésité abdominale (TT $\geq 94$ chez les hommes et TT $\geq 80 \mathrm{~cm}$ chez les femmes) (Alberti et al., 2009).

\section{Les variables indépendantes}

Les variables indépendantes sont le ratio CT/HDL-C et le HDL-C.

\section{Procédure de collecte des données}

Les mesures anthropométriques ont été prises par des enquêteurs formés. Des échantillons de sang veineux ont été prélevés le matin à jeun et conservés dans la glace puis centrifugés dans les deux heures subséquentes. Le plasma ou le sérum ont été stockés à -30 ${ }^{\circ} \mathrm{C}$. Les lipides ont été dosés au Laboratoire de Biochimie de l'Institut des Sciences Biomédicales Appliquées de Cotonou par la méthode enzymatique colorimétrique ( $\mathrm{Wu}$, 2005) en utilisant les kits de Elitech group (Sées, France).

\section{Considérations éthiques}

L'étude a été approuvée par les comités d'éthique de la Faculté de Médecine de l'Université de Montréal (Canada) et du Ministère de la Santé du Bénin. Le consentement éclairé écrit a été obtenu de chaque participant avant leur recrutement dans l'étude. 


\section{Analyse des données}

Les données ont été analysées avec le logiciel SPSS, version 20.0 (SPSS Inc., Chicago, IL). Les tests de corrélations ont été utilisés pour rechercher la relation entre les composantes du SMet. La sensibilité, spécificité, et valeurs prédictives du ratio CT/HDL-C élevé et du HDL-C bas pour le SMet ont été calculées. Les courbes Receiver Operator Characteristic (ROC) de HDL-C et de CT/HDL-C pour prédire la présence d'au moins deux composantes du SMet ont été construites et comparées entre elles en utilisant la méthode proposée par Hanley et McNeil (1983) qui tient compte de la corrélation entre les deux AUC du fait que les courbes ROC sont basées sur le même échantillon. Le niveau de signification statistique pour les tests était fixé à $\mathrm{p}<0,05$.

\section{RESULTATS}

\section{Caractéristiques sociodémographiques des sujets}

Parmi les 416 sujets, (50\% de femmes), la moyenne d'âge des hommes était $37,2 \pm$ 10,1 ans et celle des femmes était 38,9 $\pm 9,9$ ans. Les sujets résidaient en milieu rural $(31 \%)$, semi-urbain $(37,3 \%)$ et urbain $(31,7 \%)$.

\section{Prévalence des facteurs de risque cardiométabolique}

Les données sur les prévalences et les valeurs moyennes des facteurs de RCM chez les sujets ont été publiées antérieurement (Sossa et al., 2013b). En bref, les prévalences des composantes du SMet étaient, en général, plus élevées chez les femmes en comparaison aux hommes; obésité abdominale : $65.9 \%$ versus $18,8 \%,(p<0,001)$; tension artérielle élevée : $14.9 \%$ versus $11,1 \%,(\mathrm{p}=0,24)$; HDL$\mathrm{C}$ bas: $47.1 \%$ versus $28.4 \%,(\mathrm{p}<0,001)$; glycémie élevée : $17.3 \%$ versus $22,6(\mathrm{p}=0,17)$. $\mathrm{La}$ prévalence du ratio CT/HDL-C élevée était de $28.8 \%$ chez les femmes et $16.3 \%$ chez les hommes $(\mathrm{p}=0,002)$. Un effectif de 106 sujets (67 femmes et 39 hommes), soit $25,5 \%$ de l'échantillon présentaient au moins deux composantes du SMet autres que le
HDL-C faible. La prévalence du SMet chez les femmes et les hommes était de $18,8 \%$ et $9.1 \%(\mathrm{p}=0,004)$, respectivement.

Corrélation entre les composantes du syndrome métabolique

Le Tableau 1 montre que le ratio CT/HDL-C présentait des coefficients de corrélation significatifs et plus élevés pour le tour de taille, la glycémie et les triglycérides comparativement au HDL-C. La corrélation entre le HDL-C et le CT/HDL-C était de 0,60 . Les corrélations avec les tensions artérielles n'étaient pas significatives ni pour le CT/HDL-C ni pour le HDL-C isolé.

\section{Concordance entre le HDL-C bas et le CT/HDL-C élevé}

Le Tableau 2 montre la concordance entre le HDL-bas et le CT/HDL-C élevé. La statistique Kappa de mesure d'accord entre le HDL-bas et le CT/HDL-C était de 0,41. En effet, $5 \%$ des sujets avec HDL-C bas avaient un CT/HDL-C élevé et $20,2 \%$ des adultes avec un HDL-bas avaient un ratio CT/HDL-C normal.

Sensibilité, spécificité, et valeurs prédictives du CT/HDL-C élevé et du HDL-C bas pour l'existence d'au moins deux autres composantes du SMet

Dans le Tableau 3, le ratio CT/HDL-C élevé présentait les meilleures spécificités, valeurs prédictives positives et négatives mais aussi les faibles sensibilités. Le ratio CT/HDL-C présentait aussi le meilleur coefficient Q de Yule qui mesure l'intensité de son lien avec la présence d'au moins deux composantes du SMet. En revanche, le HDL$\mathrm{C}$ bas affiche les meilleures sensibilités.

\section{Comparaison des aires sous les courbes ROC de HDL-C et CT/HDL-C}

Les Figures 1 et 2 montrent que les AUC du ratio CT/HDL-C et du HDL-C pour la prédiction de l'existence d'au moins deux composantes du SMet étaient faibles $(<0,70)$. Pour le dépistage du SMet, l'AUC du CT/HDL-C est de 0,69 (IC 95\% 0,61-0,77) 
chez les femmes et 0,68 (IC 95\% 0,59-0,77) chez les hommes. L'AUC du HDL-C est de 0,45 (IC 95\% 0,37-0,53) chez les femmes et 0,40 (IC 95\% 0,30-0,44) chez les hommes. Les AUC du ratio CT/HDL-C étaient significativement plus grandes que celles de HDL-C isolé $(\mathrm{p}<0,001)$ chez les femmes comme chez les hommes. Le CT/HDL-C prédit donc mieux la présence d'au moins deux composantes du SMet que le HDL-C isolé.
Prévalence du syndrome métabolique selon des composantes HDL-C et de CT/HDL-C

Dans le Tableau 4, la prévalence du SMet était de $13,9 \%$ en utilisant la définition actuellement recommandée et de $12.3 \%$ en remplaçant le HDLC-bas par le CT/HDL-C élevé. En combinant les deux paramètres, la prévalence du SMet devient 15,3\% $(\mathrm{p}=0,56)$. Par ailleurs, parmi les 157 sujets à HDL-C bas, $33,1 \%$ ont un SMet et chez les 94 sujets à CT/HDL-C élevé, 38,3\% présentent un SMet.

Tableau 1 : Corrélation entre les composantes du syndrome métabolique.

\begin{tabular}{|c|c|c|c|c|c|c|c|}
\hline & HDL-C & CT/HDL-C & Triglycérides & Glycémie & Tour de taille & TA systolique & TA diastolique \\
\hline \multicolumn{8}{|c|}{ 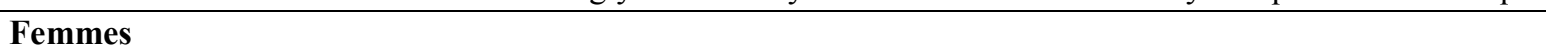 } \\
\hline HDL-C & 1 &,$- 601^{* *}$ &,$- 267^{* *}$ &,- 048 &,- 116 & 083 & ,048 \\
\hline $\mathrm{CT} / \mathrm{HDL}-\mathrm{C}$ &,$- 601^{* *}$ & 1 &, $424^{* * *}$ &, $189^{* * *}$ &, $255^{* *}$ & 075 & 105 \\
\hline Triglycérides &,$- 267^{* *}$ &, $424^{* *}$ & 1 &, 061 &, $271^{* *}$ & ,099 & $158^{*}$ \\
\hline Glycémie &,- 048 &, $189^{* *}$ & 061 & 1 & $209^{* *}$ &, $190^{* *}$ & $198^{* *}$ \\
\hline Tour de taille &,- 116 & $255^{* *}$ &, $271^{* *}$ &, $209^{* *}$ & 1 & $239^{* *}$ &, $313^{* *}$ \\
\hline TA systolique & 083 & 075 & 099 & $190^{* *}$ & $239^{* *}$ & 1 &, $774^{* *}$ \\
\hline TA diastolique & 048 & , 105 &, $158^{*}$ &, $198^{* *}$ &, $313^{* *}$ &, $774^{* *}$ & - \\
\hline \multicolumn{8}{|l|}{ Hommes } \\
\hline HDL-C & 1 &,$- 620 * *$ &,$- 156^{*}$ &,- 066 &,$- 236 * *$ & ,133 & , 121 \\
\hline CT/HDL-C &,$- 620 * *$ & 1 &, $341 * *$ & ,218** &, $424 * *$ & ,008 & ,009 \\
\hline Triglycérides &,$- 156^{*}$ &, $341 * *$ & 1 & $200 * *$ &, $315 * *$ & 074 &,- 021 \\
\hline Glycémie &,- 066 & $218^{* * *}$ &, $200 * *$ & 1 &, $258 * *$ & , 124 & 056 \\
\hline Tour de taille &,$- 236^{* *}$ &, $424 * *$ &, $315 * *$ &, $258 * *$ & 1 &, $304 * *$ & ,209** \\
\hline TA systolique & ,133 & ,008 & ,074 & , 124 &, $304 * *$ & 1 &, $795 * *$ \\
\hline TA diastolique & , 121 & 009 &,- 021 &, 056 & $209 * *$ &, $795 * *$ & 1 \\
\hline
\end{tabular}

Corrélation de Pearson ; **.P $<0,01,{ }^{*} \mathrm{P}<0,05$; HDL-C: Cholestérol HDL

CT/HDL-C: Ratio cholestérol total par cholestérol HDL; TA: Tension artérielle.

Tableau 2 : Concordance entre le CT/HDL-C élevé et le HDL-C bas.

\begin{tabular}{lcccr}
\hline & & \multicolumn{2}{c}{ CT/HDL-C (N\%) } & \multirow{2}{*}{ Total (N\%) } \\
\cline { 2 - 4 } & & Normal & Elevé & \\
\hline \multirow{2}{*}{ HDL -C } & Normal & $238(57,2)$ & $\mathbf{2 1 ( 5 , 0 )}$ & $259(62,3)$ \\
\cline { 2 - 4 } & Bas & $\mathbf{8 4}(\mathbf{2 0 , 2})$ & $73(17,5)$ & $157(37,7)$ \\
\hline Total & & $322(77,4)$ & $94(22,6)$ & $416(100)$ \\
\hline
\end{tabular}

Mesure d'accord Kappa = 0,41, p<0,001; HDL-C: Cholestérol HDL ; CT/HDL-C: Ratio cholestérol total par cholestérol HDL. 
Tableau 3: Sensibilité, spécificité, et valeurs prédictives du ratio CT/HDL-C élevé et du HDL-C bas pour l'existence d'au moins deux autres composantes du syndrome métabolique.

\begin{tabular}{|c|c|c|c|c|c|}
\hline $\begin{array}{l}\text { Facteurs de } \\
\text { risque }\end{array}$ & Sensibilité & Spécificité & $\begin{array}{c}\text { Coefficient } Q \\
\text { de Yule }\end{array}$ & VPP* & VPN** \\
\hline \multicolumn{6}{|l|}{ Femmes } \\
\hline HDL-C bas & 0,56 & 0,56 & 0,24 & 0,37 & 0,72 \\
\hline CT/HDL-C élevé & 0,47 & 0,80 & $\mathbf{0 , 5 7}$ & 0,53 & 0,76 \\
\hline \multicolumn{6}{|l|}{ Hommes } \\
\hline HDL-C bas & 0,38 & 0,79 & 0.28 & 0,25 & 0,83 \\
\hline CT/HDL-C élevé & 0,25 & 0,85 & 0,35 & 0,29 & 0,83 \\
\hline
\end{tabular}

Tableau 4 : Prévalence du syndrome métabolique en fonction de HDL-C bas et de CT/HDL-C élevé.

\begin{tabular}{|c|c|c|c|c|}
\hline & & \multicolumn{2}{|c|}{$\begin{array}{l}\text { Syndrome métabolique } \\
\text { (définition harmonisée) }\end{array}$} & \multirow[t]{2}{*}{ Total } \\
\hline & & Non $\mathbf{N}(\%)$ & Oui N(\%) & \\
\hline \multirow{2}{*}{$\begin{array}{l}\text { Syndrome métabolique (HDL- } \\
\mathrm{C} \text { bas remplacé par CT/HDL-C } \\
\text { élevé) }\end{array}$} & Oui & $6(1,4)$ & $45(10,8)$ & $51(\mathbf{1 2 , 3 )}$ \\
\hline & Non & $352(84,6)$ & $13(3,1)$ & $365(87,7)$ \\
\hline Total & & $358(86,1)$ & $58(\mathbf{1 3 , 9 )}$ & $416(100,0)$ \\
\hline
\end{tabular}

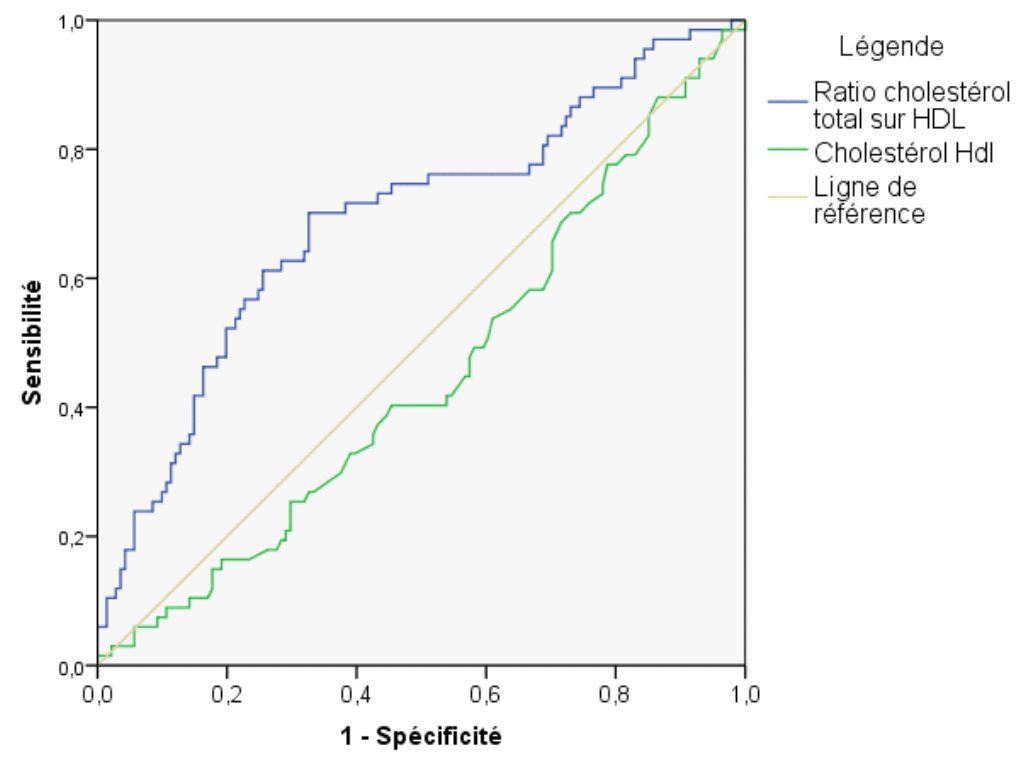

Figure 1: Comparaison des aires sous les courbes ROC prédictives d'au moins deux autres composantes du syndrome métabolique par le ratio Cholestérol Total/HDL-Cholestérol et le HDLCholestérol seul chez les femmes. 


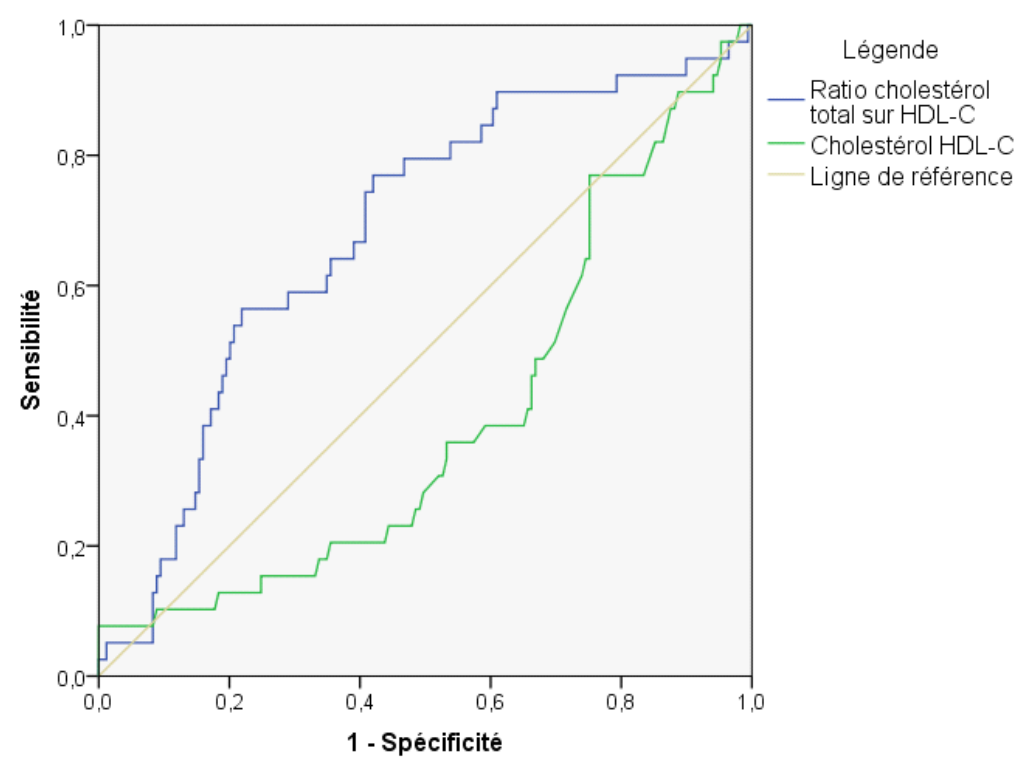

Figure 2: Comparaison des aires sous les courbes ROC prédictives d'au moins deux autres composantes du syndrome métabolique par le ratio Cholestérol Total/HDL-Cholestérol et le HDLCholestérol seul chez les hommes.

\section{DISCUSSION}

La présente étude est à notre connaissance, l'une des premières études en Afrique occidentale à explorer la force prédictive du HDL-C et le ratio CT/HDL-C pour le SMet. L'étude a permis de montrer que le HDL-C bas est plus sensible mais moins spécifique que le CT/HDL-C élevé pour l'identification des sujets présentant au moins deux autres composantes du SMet. Le CT/HDL-C a une valeur prédictive plus élevée que le HDL-C utilisé isolément.

\section{Le ratio CT/HDL-C est plus fortement corrélé aux composantes du SMet que le HDL-C}

Dans une étude antérieure (Sossa et al., 2013a), il a été rapporté que le HDL-C bas était fréquent autant chez les femmes obèses que chez les non obèses et que la distribution de cette dyslipoprotéinémie était indépendante de l'insulino-résistance. Selon Sumner et al. (2010), le HDL-C bas représente la dyslipidémie la plus répandue chez les Africains subsahariens. Cette baisse des taux de HDL-C peut être attribuée au mode de vie, l'alimentation et une espérance de vie plus longue (Windler et al., 2007).

La prévalence de HDL-C bas est élevée dans la population mais il est possible que le risque associé dépende d'autres facteurs de sorte qu'il soit en général assez bas et qu'en remplaçant le HDL-C par le ratio CT/ HDL-C, on aurait un reflet plus fidèle du risque. De plus en plus il est évoqué la notion de cholestérol HDL-C dysfonctionnel, la qualité du HDL-C prévaudrait sur la quantité. Par ailleurs, il est de plus en plus remis en question le HDL-C bas comme facteur de risque, comme en l'augmentant on ne réduit pas forcément le risque cardio-métabolique (Keene et al., 2014)

Plusieurs études ont rapporté la relation entre les facteurs de RCM et le cholestérol total à cause certainement du very low density lipoprotein et intermermediat low density lipoprotein (VLDL-C et IDL-C) que contient ce dernier. Fezeu et al. (2010) dans une étude portant sur 1471 Camerounais vivant en milieu rural et 185 Français, âgés de 25 ans et 
plus sans traitement de diabète ou d'hypertension artérielle avaient observé qu'une augmentation du tour de taille de $5 \mathrm{~cm}$ était associée à une élévation du cholestérol total chez les femmes de $0,07 \mathrm{mmol} / 1$ et chez les hommes de $0,10 \mathrm{mmol} / \mathrm{l})$. Sossa et al. (2015) avaient rapporté que le ratio $\mathrm{CT} / \mathrm{HDL}$ $\mathrm{C}$ prédit mieux l'insulino-résistance que le HDL-C isolé chez les femmes adultes. En convergence avec le résultat ci-dessus, LongoMbenza et al.(2007) dans une étude transversale portant sur la relation entre le tour de taille et le cholestérol sanguin auprès de 48 hommes et 52 femmes en Afrique centrale, ont rapporté, à partir d'un modèle multivarié, que le tour de taille était un déterminant majeur du taux de cholestérol total.

\section{Concordance entre le HDL-C bas et le CT/HDL-C élevé}

La faible concordance entre le HDL-C et le CT/HDL-C cholestérol peut s'expliquer en partie par le non HDL-C présent dans le Cholestérol total, mais aussi par les constats suivants. Chez un homme avec un taux de cholestérol total de $5,8 \mathrm{mmol} / 1$ et une concentration de HDL-C de $1,1 \mathrm{mmol} / \mathrm{l}$, on obtient un ratio $\mathrm{CT} / \mathrm{HD}-\mathrm{C}$ de 5,3 , ce qui indique un risque athérogène (Millan et al., 2009) malgré un niveau de HDL-C normal. De même, avec un niveau de cholestérol total de 4,0 mmol/l, si le taux de HDL-C était de 0,9 mmol/1, le ratio $\mathrm{CT} / \mathrm{HDL}-\mathrm{C}$ serait normal $(4,4)$. Donc, un taux normal de HDL-C peut trahir un ratio $\mathrm{CT} / \mathrm{HDL}-\mathrm{C}$ anormal comme un taux faible de HDL-C peut trahir un CT/HDLC normal.

\section{Le CT/HDL-C prédit mieux l'existence d'au moins deux composantes du SMet}

Les aires sous les courbes ROC montrent qu'aucun des deux paramètres n'a une aire suffisante $(0,7 \leq \mathrm{AUC} \leq 0,8)$ (Hosmer et Lemeshow, 2000) pour être un prédicteur acceptable de l'existence d'au moins deux composantes du SMet. Toutefois, le ratio CT/HDL-C prédit mieux l'existence d'au moins deux composantes du SMet que le HDL-C isolé.
En effet, le cholestérol HDL-C transporte le cholestérol qui efflue des macrophages vers le foie pour son élimination. Toutefois, l'étude de Khera et al. (2011) publiée récemment a montré que le taux de C-HDL, bien que corrélé avec l'efflux du cholestérol des macrophages n'était responsable de la variabilité que dans une proportion de $40 \%$. Par ailleurs des études ont montré qu'un HDL-C bas est associé à la dénutrition comme à la surnutrition (Delisle et al., 2013 )

L'observation selon laquelle le cholestérol total est un prédicteur puissant du risque coronarien a en partie justifié l'inclusion du cholestérol total dans l'équation de Framingham pour en améliorer la prédiction des risques (D'Agostino et al., 2008). Tout ceci a contribué à l'adoption du ratio $\mathrm{CT} / \mathrm{HDL}-\mathrm{C}$ par la société cardiovasculaire canadienne comme paramètre cible secondaire de suivi du traitement des dyslipidémies athérogènes (Genest et al., 2009).

Le Cholestérol total, en effet, inclut les lipoprotéines riches en triglycérides et LDLC. Le ratio $\mathrm{CT} / \mathrm{HDL}-\mathrm{C}$ a donc l'avantage d'exprimer plus convenablement la proportion entre les lipidiques athérogènes et celle anti-athérogènes de même que son inverse, le HDL-C/CT, qui indique la proportion de taux de cholestérol protecteur par rapport au reste non protecteur (Pinto et Ros, 2000) qui inclut le LDL-C qui a été le premier indice de risque de $\mathrm{MCV}$ et la principale cible pour la thérapie (Genest et al., 2009). Le ratio CT/HDL-C se révèle comme une composante mieux corrélée aux autres composantes du SMet, moins sensible et plus prédictif de l'existence d'au moins deux composantes (autres que le HDL-C) du SMet comparativement au HDL-C.

Les limites de la présente étude sont liées au fait que nous n'avons pas pris en compte dans les analyses, la modulation des facteurs tels que l'alimentation et l'activité physique sur les lipides sanguins. L'étude a été effectuée uniquement dans la partie Sud du Bénin et, par conséquent, l'extrapolation des 
résultats à d'autres groupes de population exige de la prudence.

\section{Conclusion}

Le HDL-C et le CT/HDL-C ont une faible capacité prédictive pour le SMet mais le $\mathrm{CT} / \mathrm{HDL}-\mathrm{C}$ est un meilleur prédicteur du SMet que le HDL-C utilisé isolément. Toutefois, l'utilisation de l'un ou l'autre des deux paramètres ne modifie pas substantiellement la prévalence du SMet dans la population d'étude.

\section{CONFLITS D'INTÉRÊTS}

Les auteurs ne déclarent aucun conflit d'intérêts.

\section{CONTRIBUTIONS DES AUTEURS}

HD a conçu l'étude. Charles Jérome Sossa a développé le protocole et a collecté les données sous la supervision de VA; CJS, $\mathrm{NP}, \mathrm{CSA}$ ont analysé les données et rédigé la première version du manuscrit. Tous les coauteurs ont contribué à la révision et de la finalisation du document.

\section{REMERCIEMENTS}

Nous remercions les participants de l'étude.

\section{RÉFÉRENCES}

Alberti KG, Eckel RH, Grundy SM, Zimmet PZ, Cleeman JI Donato KA, Fruchart JC, James WP, Loria CM, Smith SC. 2009. Harmonizing the metabolic syndrome: a joint interim statement of the International Diabetes Federation Task Force on Epidemiology and Prevention; National Heart, Lung, and Blood Institute; American Heart Association; World Heart Federation; International Atherosclerosis Society; and International Association for the Study of Obesity. Circulation, 120(16): 1640-1645.

D'Agostino RB, Vasan RS, Pencina MJ, Wolf PA, Cobain M, Massaro JM, Kannel WB. (2008). General cardiovascular risk profile for use in primary care: the
Framingham Heart Study. Circulation, 117(6): 743-753.

Delisle H, Ntandou G, Sodjinou R, Couillard C, Despres JP. 2013. At-risk serum cholesterol profile at both ends of the nutrition spectrum in West African adults? The Benin study. Nutrients, 5(4): 1366-1383.

Després JP, Lemieux I, Bergeron J, Pibarot P, Mathieu P, Larose E, Rodes-Cabau J, Bertrand OF, Poirier P. 2008. Abdominal obesity and the metabolic syndrome: contribution to global cardiometabolic risk. Arteriosclerosis, Thrombosis, and Vascular Biology, 28(6): 1039-1049.

Fezeu L, Balkau B, Sobngwi E, Kengne AP, Vol S, Ducimetiere P, Mbanya JC. 2010. Waist circumference and obesity-related abnormalities in French and Cameroonian adults: the role of urbanization and ethnicity. Int. J. Obes., 34(3): 446-453.

Ford ES, Giles WH, Dietz WH. (2002). Prevalence of the metabolic syndrome among US adults: findings from the third National Health and Nutrition Examination Survey. JAMA, 287(3):356359.

Genest J, McPherson R, Frohlich J, Anderson $\mathrm{T}$, Campbell N Carpentier A, Couture P, Dufour R, Fodor G, Francis GA, Grover S, Gupta M, Hegele RA, Lau DC, Leiter L, Lewis GF, Lonn E, Mancini GB, Pearson GJ, Sniderman A, Stone JA. 2009. Canadian Cardiovascular Society/Canadian guidelines for the diagnosis and treatment of dyslipidemia and prevention of cardiovascular disease in the adult - 2009 recommendations. Canadian Journal of Cardiology, 25(10): 567-579.

Hanley AJ, McNeil. 1983. A method of comparing the areas under Receiver Operating Curves derived from the same cases. Radiology, 148(3): 839-843.

Hosmer D, Lemeshow S. 2000. Applied Logistic Regression (2nd edn). John Wiley \& Sons, Inc: New York.

Keene D, Shun-Shin M, Price C. 2014. The Observational HDL hypothesis: A useful 
therapeutic target? A meta analysis of 117,411patients in randomised controlled trials. Heart, 100: A62-A63.

Khera AV, Cuchel M, de la Llera-Moya M, Rodrigues A, Burke MF, Jafri K, French BC, Phillips JA, Mucksavage ML, Wilensky R.L, Mohler ER, Rothblat GH, Rader DJ. 2011. Cholesterol efflux capacity, high-density lipoprotein function, and atherosclerosis. New England Journal of Medicine, 364(2): 127-135.

Longo-Mbenza B, Mambune HF, Kasiam JB, Vita EK, Fuele SM Nsenga JN, Mabwa L, Nzuzi V. 2007. Relationship between waist circumference and cholesterol in Central Africans with congestive heart failure. West African Journal of Medicine, 26(3): 183-190.

Millan J, Pinto X, Munoz A, Zuniga M, Rubies-Prat J Pallardo LF, Massana L, Mangas A, Hernandez Mijares A, Gonzalez-Santos P, Ascaso JF, PedroBotet J. 2009. Lipoprotein ratios: Physiological significance and clinical usefulness in cardiovascular prevention. Vasc. Health Risk Manag, 5(7): 757-765.

Ntandou G, Delisle H, Agueh V Fayomi B. 2009. Abdominal obesity explains the positive rural-urban gradient in the prevalence of the metabolic syndrome in Benin, West Africa. Nutr. Res., 29(3): 180-189.

Pintó X, Ros E. 2000. Lípidos séricos y predicción del riesgo cardiovascular: importancia de los cocientes colesterol total/colesterol HDL y colesterol LDL/colesterol HDL. Clin Invest Arterioscl., 12: 267-284.

Sossa C, Delisle H, Agueh V, Makoutode M Fayomi B. 2013a. Insulin resistance status and four-year changes in other cardiometabolic risk factors in West-
African adults: the Benin study. Eur. J. Prev. Cardiol., 20(6): 1042-1050.

Sossa C, Delisle H, Agueh V, Sodjinou R, Ntandou G, Makoutodé M. 2013b. Lifestyle and dietary factors associated with the evolution of cardiometabolic risk over four years in West-African adults: the Benin study. J. Obes., 2013: 9p.

Sossa C, Delisle H, Agueh V, Saizonou J, Paraiso MN, Kpozehouen A, Makoutodé M, Delisle H. 2015. Prédiction de l'insulino-résistance par les ratios de lipoprotéines chez les adultes béninois Int. J. Biol. Chem. Sci., 9(3): 1319-1329.

Sumner AE. 2009. Ethnic differences in triglyceride levels and high-density lipoprotein lead to underdiagnosis of the metabolic syndrome in black children and adults. J. Pediatr., 155(3): S7.e7-11.

Sumner AE, Zhou J, Doumatey A, Imoisili OE, Amoah A, Acheampong J, Oli J, Johnson T, Adebamowo C, Rotimi CN. 2010. Low HDL-Cholesterol with Normal Triglyceride Levels is the Most Common Lipid Pattern in West Africans and African Americans with Metabolic Syndrome: Implications for Cardiovascular Disease Prevention. C.V.D. Prev. Control, 5(3): 75-80.

Windler E, Schoffauer M Zyriax BC. 2007. The significance of low HDL-cholesterol levels in an ageing society at increased risk for cardiovascular disease. Diab. Vasc. Dis. Res., 4(2): 136-142.

$\mathrm{Wu}$ A. (2005). Tielz Clinical Guide to Laboratory Tests (fourth edition). W.B. Saunders Company: Philadelphia. 